\title{
Avaliação do contato de dois materiais retrobturadores na densidade óptica de corantes tamponados e não tamponados
}

\author{
Evaluation of the contact of two retrofilling materials on optical density of buffered \\ and unbuffered dyes
}

\author{
Neuza Maria Souza Picorelli ASSIS \\ Professora - Departamento de Clínica Odontológica - Disciplina de Anestesiologia em Odontologia e Cirurgia \\ Maxilofacial I, II e III - Faculdade de Odontologia de Juiz de Fora, Universidade Federal de Juiz de Fora - MG \\ Brasil - Doutoranda - Programa de Pós-Graduação em Odontologia Restauradora - Departamento de Odontologia \\ Restauradora - Faculdade de Odontologia de São José dos Campos - Universidade Estadual Paulista - UNESP - \\ São José dos Campos - SP - Brasil
}

\section{Ana Paula Martins GOMES}

Professora Adjunta - Departamento de Odontologia Restauradora - Faculdade de Odontologia de São José dos Campos - Universidade Estadual Paulista - UNESP - São José dos Campos - SP - Brasil

\section{Claudio Hideki KUBO}

Doutorando - Programa de Pós-Graduação em Odontologia Restauradora - Departamento de Odontologia

Restauradora - Faculdade de Odontologia de São José dos Campos - Universidade Estadual Paulista - UNESP São José dos Campos - SP - Brasil

\section{Maria Nadir Gasparoto MANCINI}

Professora Assistente Doutora - Departamento de Biociências e Diagnóstico Bucal - Faculdade de Odontologia de São José dos Campos - Universidade Estadual Paulista - UNESP - São José dos Campos - SP - Brasil

\begin{abstract}
Resumo
O objetivo deste trabalho foi avaliar a densidade óptica das soluções corantes azul de metileno $2 \%$ tamponada e não tamponada, rodamina B 2\% tamponada e não tamponada, antes e após a imersão de dois materiais retrobturadores por diferentes períodos de tempo. As soluções corantes, sem a imersão dos espécimes, foram analisadas nos períodos de 0 , 12, 24, 48 e 72 horas. Foram preparados oitenta espécimes de cimento Sealer 26 e oitenta de cimento de Portland, os quais foram imersos em $0,7 \mathrm{ml}$ de cada uma das soluções corantes e avaliados nos períodos de tempo de 12, 24, 48 e 72 horas. A densidade óptica foi avaliada em espectrofotômetro ajustado em 596nm para o azul de metileno e 566nm para a rodamina B. Os dados obtidos foram analisados através dos testes ANOVA (3 fatores) e Tukey 5\%. Os resultados mostraram que houve diferença estatisticamente significativa nos valores da densidade óptica do azul de metileno $2 \%$ tamponado após a imersão do Sealer 26 nos períodos de 24, 48 e 72 horas em relação ao tempo de 12 horas. Houve diferença estatisticamente significativa nos valores da densidade óptica da rodamina B 2\% tamponada após a imersão do cimento de Portland nos períodos de 12, 24 e 48 horas em relação ao tempo de 72 horas. Pôde-se verificar que as soluções corantes não tamponadas apresentaram valores menores de densidade óptica. Dentre as soluções corantes analisadas, a rodamina B $2 \%$ tamponada apresentou comportamento mais estável quanto aos valores de densidade óptica até o período de avaliação de 48 horas.
\end{abstract}

\section{UNITERMOS}

Corante; densidade óptica; materiais dentários; material retrobturador. 


\section{INTRODUÇÃO}

O objetivo do tratamento endodôntico é a limpeza, instrumentação e obturação dos canais radiculares, prevenindo a proliferação de microrganismos e seus sub-produtos. Nos casos de insucesso, se o retratamento endodôntico fracassar ou estiver contra-indicado, a cirurgia parendodôntica deve ser realizada como alternativa para se obter o sucesso ${ }^{8,12}$.

A maioria dos materiais empregados na cirurgias parendodônticas passou a ser utilizada na Endodontia a partir da Odontologia Restauradora, sem levar em consideração as exigências diferentes e condições especiais do ápice radicular ${ }^{1}$. Para atender a estas exigências, um material experimental denominado Agregado de Trióxido Mineral (MTA) tem sido investigado como material alternativo em Endodontia, podendo ser utilizado em retrobturações de canais radiculares ${ }^{1,19,20,21}$. O MTA foi desenvolvido na Universidade de Loma Linda pelo Dr. Mahmoud Torabinejad e seus colaboradores, com a finalidade de selar as vias de comunicação entre o sistema de canais e a superfície externa do dente. Foi lançado no mercado com o nome de Pro Root - MTA (Denstply, Tulsa Dental, Tulsa, Oklahoma, USA).

Alguns estudos ${ }^{7,22}$ verificaram que o MTA (Pro Root) apresentava características macroscópicas, microscópicas e comportamento biológico semelhantes ao cimento de Portland. Estrela et al. ${ }^{7}$ (2000) constataram que a composição, a ação antimicrobiana e o pH entre o MTA (Pro Root) e o cimento de Portland eram semelhantes, com a exceção de que o cimento de Portland não apresentava o óxido de bismuto, que é o radiopacificador do Pro Root. Uma firma nacional (Angelus) purificou e colocou radiopacificador no cimento de Portland, lançando um MTA nacional no mercado ${ }^{6}$.

Alguns estudos comparando a biocompatibilidade do MTA e do cimento de Portland foram realizados in vivo e in vitro e os resultados sugeriram que o cimento de Portland tem potencial para ser utilizado como material retrobturador com custo mais acessível ${ }^{2,4}$.

O Sealer 26 é um cimento endodôntico derivado do AH26, que possui em sua composição hidróxido de cálcio e óxido de bismuto aglutinado por resina epóxica. Apresenta boa capacidade seladora como material obturador de canais radiculares e também como material retrobturador ${ }^{12,18}$.

A avaliação do selamento apical utilizando corantes é um método largamente empregado para avaliar materiais retrobturadores ${ }^{3,9,19}$. Entretanto, o corante pode interagir quimicamente com o material de selamento ou a dentina e esta interação pode influenciar a difusão ou a descoloração do traçador, impedindo a adequada avaliação da infiltração marginal ${ }^{14,23,24,25}$. Souza ${ }^{16}(2004)$ avaliou a infiltração apical do azul de metileno a $2 \%$ e da rodamina B $2 \%$ em função da composição química do cimento endodôntico utilizado para a obturação dos canais radiculares. $\mathrm{O}$ autor verificou que o corante azul de metileno, quando comparado à rodamina $\mathrm{B}$, apresentou menores níveis de infiltração nos cimentos Endofill, Sealer 26 e Sealapex, sugerindo uma incapacidade da solução de azul de metileno a $2 \%$ em revelar a totalidade da falha da obturação realizada com estes cimentos. $\mathrm{Kubo}^{11}$ (2001) avaliou a infiltração da rodamina B $2 \%$ preparada a partir de tampão fosfato ajustado com $\mathrm{pH}$ sete, em retrobturações realizadas com Pro Root-MTA. $\mathrm{O}$ autor verificou a penetração do corante na interface dente material retrobturador além da metade da profundidade da retrobturação.

Até o momento, não foi totalmente estabelecido se o contato dos materiais retrobturadores com as soluções corantes pode produzir reações que levem a modificações nestes traçadores. Para tanto, o objetivo do presente estudo foi avaliar a densidade óptica de quatro soluções corantes antes e após a imersão de dois materiais por diferentes períodos de tempo.

\section{Material e método}

As quatro soluções corantes foram preparadas no mesmo período, permanecendo acondicionadas em frasco de vidro âmbar em temperatura ambiente $\left(23 \pm 3^{\circ} \mathrm{C}\right)$.

A solução de azul de metileno $2 \%$ não tamponada foi preparada dissolvendo-se $2 \mathrm{~g}$ de azul de metileno (Synth, Labsynth Produtos para Laboratórios Ltda, Diadema, São Paulo, Brasil) em 100ml de água destilada. A solução de azul de metileno $2 \%$ tamponada em tampão fosfato $\mathrm{pH}$ 7,0 foi preparada dissolvendo-se $2 \mathrm{~g}$ de azul de metileno (Synth, Labsynth Produtos para Laboratórios Ltda, Diadema, São Paulo, Brasil) em $100 \mathrm{ml}$ de tampão fosfato $0,2 \mathrm{M} \mathrm{e} \mathrm{pH} 7,0$ a $37^{\circ} \mathrm{C}$.

A solução de rodamina B $2 \%$ não tamponada foi preparada dissolvendo-se $2 \mathrm{~g}$ de corante rodamina $\mathrm{B}$ (Synth, Labsynth Produtos para Laboratórios Ltda, Diadema, São Paulo, Brasil) em $100 \mathrm{ml}$ de água destilada. A solução de rodamina B $2 \%$ tamponada em tampão fosfato $\mathrm{pH}$ 7,0 foi preparada dissolvendo-se $2 \mathrm{~g}$ de corante rodamina B (Synth, Labsynth Produtos para Laboratórios Ltda, Diadema, São Paulo, Brasil) em $100 \mathrm{ml}$ de tampão fosfato $0,2 \mathrm{M}$ e pH 7,0 a $37^{\circ} \mathrm{C}$. 
Foram preparados 160 espécimes, sendo oitenta do cimento Sealer 26 (Dentsply Ind. e Comércio, Rio de Janeiro, RJ, Brasil) e oitenta do cimento Portland (Votorantim, Brasil). Os espécimes do cimento Sealer 26 foram manipulados na proporção de $0,24 \mathrm{~g}$ de pó para $0,064 \mathrm{~g}$ de resina ${ }^{18}$. Os espécimes do cimento Portland foram manipulados na proporção $1 \mathrm{~g}$ de pó para $0,33 \mathrm{~g}$ de água destilada ${ }^{20}$. Para padronização dos espécimes, foi preparada uma matriz de silicona borrachóide (Silibidor, Clássico, São Paulo, Brasil). Após a manipulação, o material foi inserido na matriz com auxílio de um calcador (S.S. White 6335, \#1 Ref. 10455, S.S. White, Rio de Janeiro, RJ, Brasil). Os excessos foram removidos com auxílio de gaze. Todos os espécimes apresentaram forma cilíndrica com $2 \mathrm{~mm}$ de altura e $3 \mathrm{~mm}$ de diâmetro. Para assegurar o endurecimento dos materiais sob condição de umidade e temperatura padronizadas, a matriz preenchida com os espécimes do cimento Sealer 26 ou Portland, foi mantida sob condições de umidade relativa, por 24 horas a $37 \pm 1^{\circ} \mathrm{C}$ (Estufa tipo forno Pasteur, Fabbe Primar Industrial Ltda. do laboratório de Microbiologia da FOSJC). Após o período de 24 horas, os espécimes foram removidos da matriz. Apenas os espécimes padronizados, com as dimensões confirmadas por um paquímetro digital (Starrett, cat. No. 727- 6/150, Starrett Indústria e Comércio Ltda., Itu, SP, Brasil), foram utilizados no trabalho.

O estudo da densidade óptica das soluções corantes foi dividido em duas partes: análise das soluções corantes sem a imersão dos espécimes; e análise das soluções corantes após a imersão dos espécimes por $12,24,48$ ou 72 horas. O estudo da densidade óptica das quatro soluções corantes, sem a imersão de qualquer espécime, foi realizado em dois períodos distintos (período 1 e período 2), e simultaneamente ao período em que foi realizada a análise das soluções corantes após a imersão dos espécimes de cada material. $\mathrm{Ou}$ seja, as análises das soluções no período 1 (sem imersão de espécime) foram realizadas durante a avaliação das soluções após a imersão do cimento Sealer 26 e as análises das soluções no período 2 (sem imersão de espécime) foram realizadas durante a avaliação das soluções após a imersão do cimento de Portland. A densidade óptica foi analisada em duplicata nos períodos de $0,12,24,48$ e 72 horas.

Análise das soluções corantes após a imersão dos espécimes por 12, 24, 48 ou 72 horas

Oitenta espécimes de cada material foram inseridos, individualmente, em recipientes de vidro de 20ml na cor âmbar, com identificação na tampa. Os recipientes apresentavam volume interno que permitia a imersão do espécime em $0,7 \mathrm{ml}$ da solução corante, pipetada com pipeta automática (PIPETMAN P100, Gilson, França). Os conjuntos formados por oitenta espécimes de cada material, inseridos em oitenta recipientes, foram aleatoriamente divididos de acordo com a solução corante em quatro grupos:

a) grupo 1: vinte espécimes imersos na solução de azul de metileno $2 \%$ tamponada;

b) grupo 2: vinte espécimes imersos na solução de azul de metileno $2 \%$ não tamponada;

c) grupo 3: vinte espécimes imersos na solução de rodamina B $2 \%$ tamponada;

d) grupo 4: vinte espécimes imersos na solução de rodamina $\mathrm{B} 2 \%$ não tamponada.

Após a imersão dos espécimes nas soluções corantes, cada grupo experimental foi subdividido de acordo com o tempo que cada espécime permaneceu imerso na solução a temperatura ambiente $\left(23 \pm 3^{\circ} \mathrm{C}\right)$, em quatro subgrupos (SG), com cinco espécimes de cada material imerso na solução corante por 12 horas (SG A); 24 horas (SG B); 48 horas (SG C); e 72 horas (SG D).

A densidade óptica das soluções corantes foi avaliada após 12, 24, 48 ou 72 horas de imersão dos espécimes.

\section{Análise da densidade óptica das soluções co- rantes}

Os comprimentos de onda de absorção máxima dos corantes foram determinados através do espectro de varredura no intervalo de 100 a $1000 \mathrm{~nm}$ em espectrofotômetro Shimadzu UV (série 1203, Kyoto, Japão) (Figura 1). A solução do corante azul de metileno $2 \%$, tamponada ou não, apresentou pico máximo de absorção em 596nm. A solução do corante rodamina B 2\%, tamponada ou não, apresentou pico máximo de absorção em 566nm.

Para a determinação da densidade óptica de cada réplica da condição experimental, removeu-se do frasco de vidro âmbar uma alíquota de $0,1 \mathrm{ml}$ de solução corante, que foi transferida para um tubo de ensaio, diluída em 1,9ml de água deionizada e agitada levemente para homogeneização. A seguir, $1,5 \mathrm{ml}$ da solução corante foi inserida em uma cubeta para análise no espectrofotômetro. Para as soluções de azul de metileno e rodamina $\mathrm{B}$, tamponadas ou não, foram empregados os comprimentos de $596 \mathrm{~nm}$ e 566nm, respectivamente. As densidades óticas das quatro soluções corantes, sem a imersão de qualquer 
espécime, foram analisadas por $0,12,24,48$ e 72 horas. Após a imersão dos espécimes de Sealer 26 ou cimento de Portland, a densidade óptica das quatro soluções corantes foi analisada nos períodos de 12 , 24,48 e 72 horas.

A análise estatística foi dividida em análise dos dados das soluções corantes sem a imersão de qualquer espécime e após a imersão dos espécimes, nos diferentes períodos de tempo. Os dados da densidade óptica

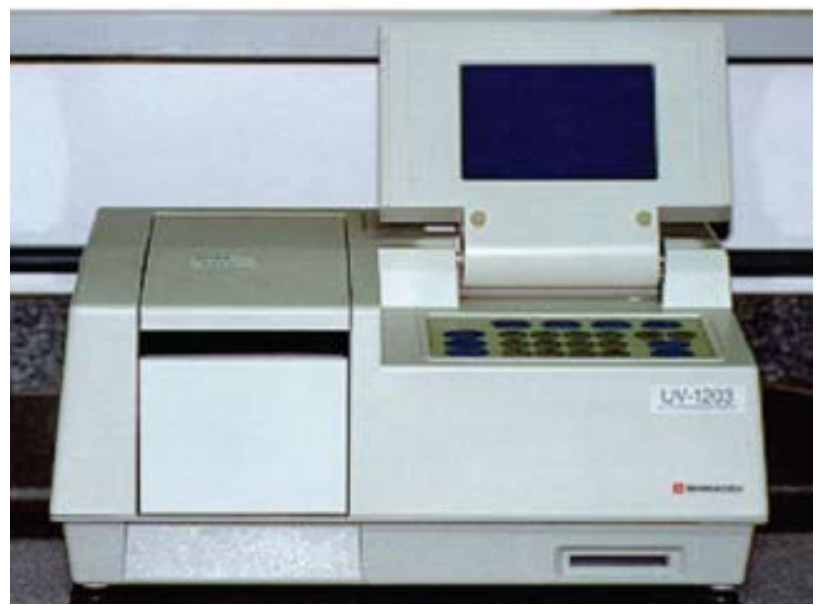

FIGURA 1 - Espectrofotômetro utilizado para a avaliação da densidade óptica das soluções corantes. foram apresentados através da estatística descritiva com os valores da média e desvio padrão, e foram submetidos à análise, numa abordagem paramétrica, através da análise de variância (ANOVA) e teste de comparação múltipla de Tukey. O nível de significância adotado foi o valor convencional de 5\%. A análise estatística dos dados e a confecção dos gráficos foram efetuadas por meio do programa computacional STATISTICA (2000, versão 5.5, Stasoft Inc.).

\section{Resultados}

Análise da densidade óptica das soluções corantes sem a imersão dos espécimes
Na Tabela 1 pode-se verificar os valores da densidade óptica nos dois períodos de análise (período 1 e 2).

Tabela 1 - Média e desvio padrão da densidade óptica verificada nos dois períodos de análise (1 e 2), pelas diferentes soluções corantes, sem a imersão de qualquer espécime

\begin{tabular}{|c|c|c|c|c|c|c|c|c|c|c|}
\hline \multirow{4}{*}{ Soluções corantes } & \multicolumn{10}{|c|}{ Sem imersão de espécimes } \\
\hline & \multicolumn{5}{|c|}{ Período 1} & \multicolumn{5}{|c|}{ Período 2} \\
\hline & \multicolumn{5}{|c|}{ Tempo (horas) } & \multicolumn{5}{|c|}{ Tempo (horas) } \\
\hline & 0 & 12 & 24 & 48 & 72 & 0 & 12 & 24 & 48 & 72 \\
\hline $\begin{array}{l}\text { Azul de Metileno } 2 \% \\
\text { tamponada }\end{array}$ & $\begin{array}{c}0,993 \\
\pm \\
0,030\end{array}$ & $\begin{array}{c}0,981 \\
\pm \\
0,013\end{array}$ & $\begin{array}{c}0,983 \\
\pm \\
0,019\end{array}$ & $\begin{array}{c}0,992 \\
\pm \\
0,020\end{array}$ & $\begin{array}{c}0,993 \\
\pm \\
0,001\end{array}$ & $\begin{array}{c}0,981 \\
\pm \\
0,013\end{array}$ & $\begin{array}{c}0,983 \\
\pm \\
0,019\end{array}$ & $\begin{array}{c}0,993 \\
\pm \\
0,030\end{array}$ & $\begin{array}{c}0,991 \\
\pm \\
0,008\end{array}$ & $\begin{array}{c}1,020 \\
\pm \\
0,003\end{array}$ \\
\hline $\begin{array}{l}\text { Azul de Metileno } 2 \% \\
\text { não tamponada }\end{array}$ & $\begin{array}{c}0,954 \\
\pm \\
0,065\end{array}$ & $\begin{array}{c}0,975 \\
\pm \\
0,008\end{array}$ & $\begin{array}{c}0,959 \\
\pm \\
0,006\end{array}$ & $\begin{array}{c}0,939 \\
\pm \\
0,018\end{array}$ & $\begin{array}{c}0,971 \\
\pm \\
0,017\end{array}$ & $\begin{array}{c}0,975 \\
\pm \\
0,008\end{array}$ & $\begin{array}{c}0,959 \\
\pm \\
0,006\end{array}$ & $\begin{array}{c}0,954 \\
\pm \\
0,065\end{array}$ & $\begin{array}{c}0,926 \\
\pm \\
0,004\end{array}$ & $\begin{array}{c}0,998 \\
\pm \\
0,002\end{array}$ \\
\hline Rodamina B $2 \%$ tamponada & $\begin{array}{c}0,979 \\
\pm \\
0,014\end{array}$ & $\begin{array}{c}0,917 \\
\pm \\
0,007\end{array}$ & $\begin{array}{c}0,929 \\
\pm \\
0,011\end{array}$ & $\begin{array}{c}0,930 \\
\pm \\
0,016\end{array}$ & $\begin{array}{c}0,934 \\
\pm \\
0,015\end{array}$ & $\begin{array}{c}0,917 \\
\pm \\
0,007\end{array}$ & $\begin{array}{c}0,929 \\
\pm \\
0,011\end{array}$ & $\begin{array}{c}0,979 \\
\pm \\
0,014\end{array}$ & $\begin{array}{c}0,941 \\
\pm \\
0,030\end{array}$ & $\begin{array}{c}0,914 \\
\pm \\
0,022\end{array}$ \\
\hline $\begin{array}{l}\text { Rodamina B } 2 \% \\
\text { não tamponada }\end{array}$ & $\begin{array}{c}0,975 \\
\pm \\
0,016\end{array}$ & $\begin{array}{c}0,956 \\
\pm \\
0,010\end{array}$ & $\begin{array}{c}0,948 \\
\pm \\
0,017\end{array}$ & $\begin{array}{c}0,959 \\
\pm \\
0,022\end{array}$ & $\begin{array}{c}0,911 \\
\pm \\
0,006\end{array}$ & $\begin{array}{c}0,956 \\
\pm \\
0,010\end{array}$ & $\begin{array}{c}0,948 \\
\pm \\
0,017\end{array}$ & $\begin{array}{c}0,975 \\
\pm \\
0,016\end{array}$ & $\begin{array}{c}0,932 \\
\pm \\
0,004\end{array}$ & $\begin{array}{c}0,949 \\
\pm \\
0,011\end{array}$ \\
\hline
\end{tabular}


Análise da densidade óptica das soluções corantes após a imersão dos espécimes

$\mathrm{Na}$ Tabela 2 e Figura 2 foram apresentados os dados da densidade óptica das soluções sem a imer- são dos espécimes (tempo zero de cada solução nos períodos 1 e 2), bem como os dados após a imersão dos espécimes do cimento Sealer 26 e cimento de Portland.

Tabela 2 - Média e desvio padrão dos valores da densidade óptica verificados sem e após a imersão dos espécimes de Sealer 26 e cimento de Portland, nos diferentes períodos de análise

\begin{tabular}{|c|c|c|c|c|c|c|c|c|c|c|}
\hline \multirow{4}{*}{ Soluções corantes } & \multicolumn{10}{|c|}{ Material } \\
\hline & \multicolumn{5}{|c|}{ Sealer 26} & \multicolumn{5}{|c|}{ Cimento de Portland } \\
\hline & \multicolumn{5}{|c|}{ Tempo (horas) } & \multicolumn{5}{|c|}{ Tempo (horas) } \\
\hline & $\begin{array}{l}\text { Sem } \\
\text { imersão }\end{array}$ & 12 & 24 & 48 & 72 & $\begin{array}{l}\text { Sem } \\
\text { imersão }\end{array}$ & 12 & 24 & 48 & 72 \\
\hline $\begin{array}{l}\text { Azul de Metileno } 2 \% \\
\text { tamponada }\end{array}$ & $\begin{array}{l}0,993 \\
\pm \\
0,030\end{array}$ & $\begin{array}{l}0,858 \\
\pm \\
0,019\end{array}$ & $\begin{array}{l}0,974 \\
\pm \\
0,026\end{array}$ & $\begin{array}{l}1,031 \\
\pm \\
0,026\end{array}$ & $\begin{array}{l}1,031 \\
\pm \\
0,025\end{array}$ & $\begin{array}{l}0,981 \\
\pm \\
0,013\end{array}$ & $\begin{array}{l}1,004 \\
\pm \\
0,023\end{array}$ & $\begin{array}{l}1,035 \\
\pm \\
0,035\end{array}$ & $\begin{array}{l}1,044 \\
\pm \\
0,033\end{array}$ & $\begin{array}{l}1,056 \\
\pm \\
0,021\end{array}$ \\
\hline $\begin{array}{l}\text { Azul de Metileno } 2 \% \\
\text { não tamponada }\end{array}$ & $\begin{array}{l}0,954 \\
\pm \\
0,065\end{array}$ & $\begin{array}{l}0,892 \\
\pm \\
0,063\end{array}$ & $\begin{array}{l}0,914 \\
\pm \\
0,018\end{array}$ & $\begin{array}{l}0,871 \\
\pm \\
0,024\end{array}$ & $\begin{array}{l}0,873 \\
\pm \\
0,011\end{array}$ & $\begin{array}{l}0,975 \\
\pm 0,008\end{array}$ & $\begin{array}{l}0,891 \\
\pm \\
0,035\end{array}$ & $\begin{array}{l}0,903 \\
\pm \\
0,034\end{array}$ & $\begin{array}{l}0,890 \\
\pm \\
0,015\end{array}$ & $\begin{array}{l}0,894 \\
\pm \\
0,007\end{array}$ \\
\hline Rodamina B $2 \%$ tamponada & $\begin{array}{l}0,979 \\
\pm \\
0,014\end{array}$ & $\begin{array}{l}0,952 \\
\pm \\
0,034\end{array}$ & $\begin{array}{l}0,938 \\
\pm \\
0,019\end{array}$ & $\begin{array}{l}0,978 \\
\pm \\
0,027\end{array}$ & $\begin{array}{l}0,945 \\
\pm \\
0,019\end{array}$ & $\begin{array}{l}0,917 \\
\pm \\
0,007\end{array}$ & $\begin{array}{l}0,952 \\
\pm \\
0,049\end{array}$ & $\begin{array}{l}0,945 \\
\pm \\
0,026\end{array}$ & $\begin{array}{l}0,951 \\
\pm \\
0,020\end{array}$ & $\begin{array}{l}1,034 \\
\pm \\
0,018\end{array}$ \\
\hline $\begin{array}{l}\text { Rodamina B } 2 \% \\
\text { não tamponada }\end{array}$ & $\begin{array}{l}0,975 \\
\pm \\
0,016\end{array}$ & $\begin{array}{l}0,870 \\
\pm \\
0,025\end{array}$ & $\begin{array}{l}0,906 \\
\pm \\
0,027\end{array}$ & $\begin{array}{l}0,871 \\
\pm \\
0,011\end{array}$ & $\begin{array}{l}0,880 \\
\pm \\
0,012\end{array}$ & $\begin{array}{l}0,956 \\
\pm \\
0,010\end{array}$ & $\begin{array}{l}0,936 \\
\pm \\
0,016\end{array}$ & $\begin{array}{l}0,915 \\
\pm \\
0,043\end{array}$ & $\begin{array}{l}0,880 \\
\pm \\
0,011\end{array}$ & $\begin{array}{l}0,885 \\
\pm \\
0,017\end{array}$ \\
\hline
\end{tabular}

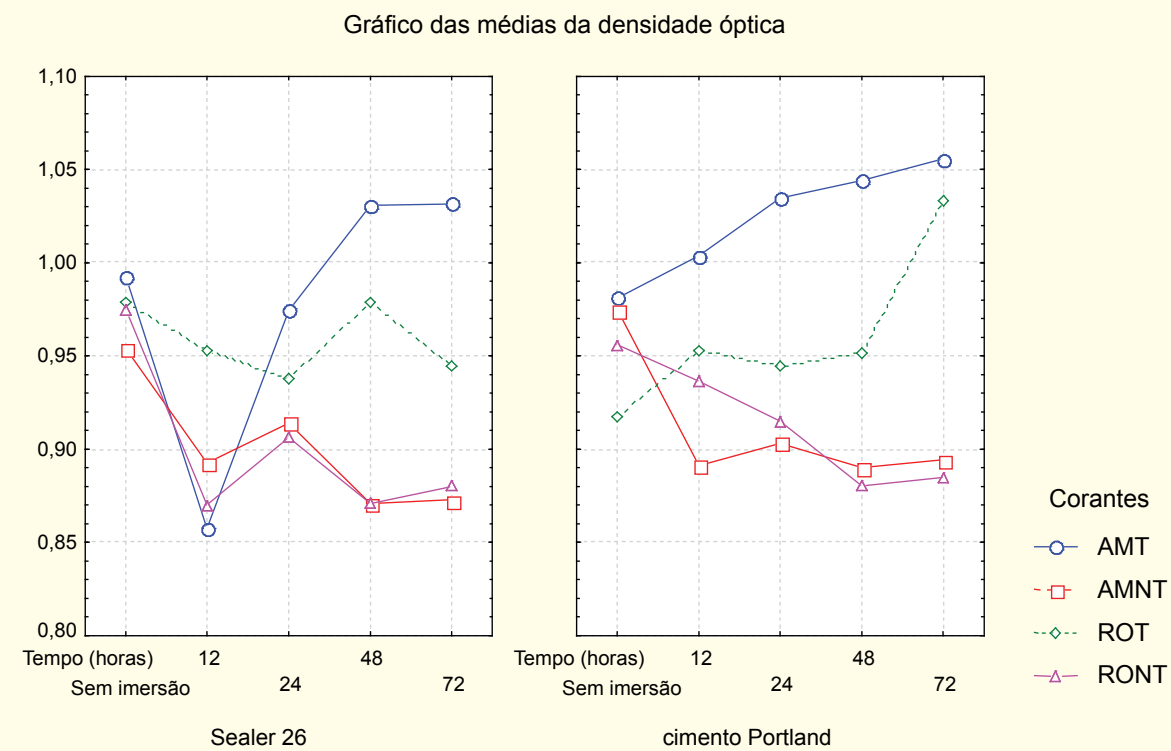

FIGURA 2 - Gráfico das médias da densidade óptica das diferentes soluções corantes sem e após a imersão dos espécimes de Sealer 26 ou cimento de Portland. 
Na Figura 2 verifica-se que o valor médio da densidade óptica após 12 horas de imersão dos espécimes de cimento Sealer 26 na solução de azul de metileno $2 \%$ tamponada foi o menor valor observado. Houve diferença estatisticamente significativa quando este valor foi comparado aos demais períodos de tempo de observação (Tabela 4). Após 24,48 e 72 horas, a solução de azul de metileno $2 \%$ tamponada apresentou os maiores valores de densidade óptica em relação aos demais corantes. A imersão dos espécimes nas soluções corantes azul de metileno $2 \%$ não tamponado, rodamina $\mathrm{B}$ $2 \%$ tamponada e não tamponada por 12, 24, 48 e 72 horas não promoveu alterações significativas nos valores da densidade óptica (Tabela 4).
A imersão dos espécimes de cimento Portland nas soluções corantes de azul de metileno $2 \%$ tamponada ou não, rodamina B $2 \%$ não tamponada por $12,24,48$ e 72 horas não promoveu alterações significativas nos valores da densidade óptica (Tabela 5). A imersão dos espécimes na solução de rodamina B 2\% tamponada promoveu aumento da densidade óptica, com diferença estatisticamente significativa apenas após 72 horas, quando comparado aos demais períodos de tempo de observação (Tabela 5).

Foram testadas as seguintes hipóteses em relação aos dados da densidade óptica: ausência de qualquer nível de interação; igualdade quanto aos materiais; igualdade quanto ao efeito do tipo de solução corante e igualdade quanto aos períodos de tempo de imersão (Tabela 3).

Tabela 3 - Resultados da ANOVA para os dados obtidos

\begin{tabular}{|c|c|c|c|c|c|}
\hline Efeito & gl & SQ & QM & $F$ & $p$ - valor \\
\hline Material (M) & 1 & $0,02894^{*}$ & $0,02894^{*}$ & $39,13^{*}$ & $0,0000^{*}$ \\
\hline Corante (C) & 3 & $0,36673^{*}$ & $0,12224^{\star}$ & $165,25^{\star}$ & $0,0000^{*}$ \\
\hline Tempo (T) & 3 & $0,01955^{\star}$ & $0,00652^{*}$ & $8,81^{*}$ & $0,0000^{*}$ \\
\hline$(\mathrm{M}) \times(\mathrm{C})$ & 3 & $0,01671^{*}$ & $0,00557^{*}$ & $7,53^{\star}$ & $0,0001^{*}$ \\
\hline$(\mathrm{C}) \times(\mathrm{T})$ & 9 & $0,08642^{*}$ & $0,00960^{\star}$ & $12,98^{\star}$ & $0,0000^{*}$ \\
\hline$(\mathrm{M}) \times(\mathrm{T})$ & 3 & $0,01392^{*}$ & $0,00464^{*}$ & $6,27^{*}$ & $0,0005^{\star}$ \\
\hline$(\mathrm{M}) \times(\mathrm{C}) \times(\mathrm{T})$ & 9 & $0,04043^{*}$ & $0,00449^{\star}$ & $6,07^{*}$ & $0,0000^{*}$ \\
\hline Resíduo & 128 & 0,09469 & 0,00074 & & \\
\hline Total & 159 & 0,66740 & & & \\
\hline
\end{tabular}

$* \mathrm{p}<0,05$

Após a análise de variância e por meio do teste de comparação múltipla de Tukey foram estabelecidos seis conjuntos de mesmo desempenho representados pelas letras A, B, C, D, E e F em termos de densidade óptica, para cada material de imersão, conforme Tabelas 4 e 5. 
Tabela 4 - Teste de comparação múltipla de Tukey (5\%) para as diferentes condições experimentais dos dados da densidade óptica após a imersão dos espécimes do cimento Sealer 26

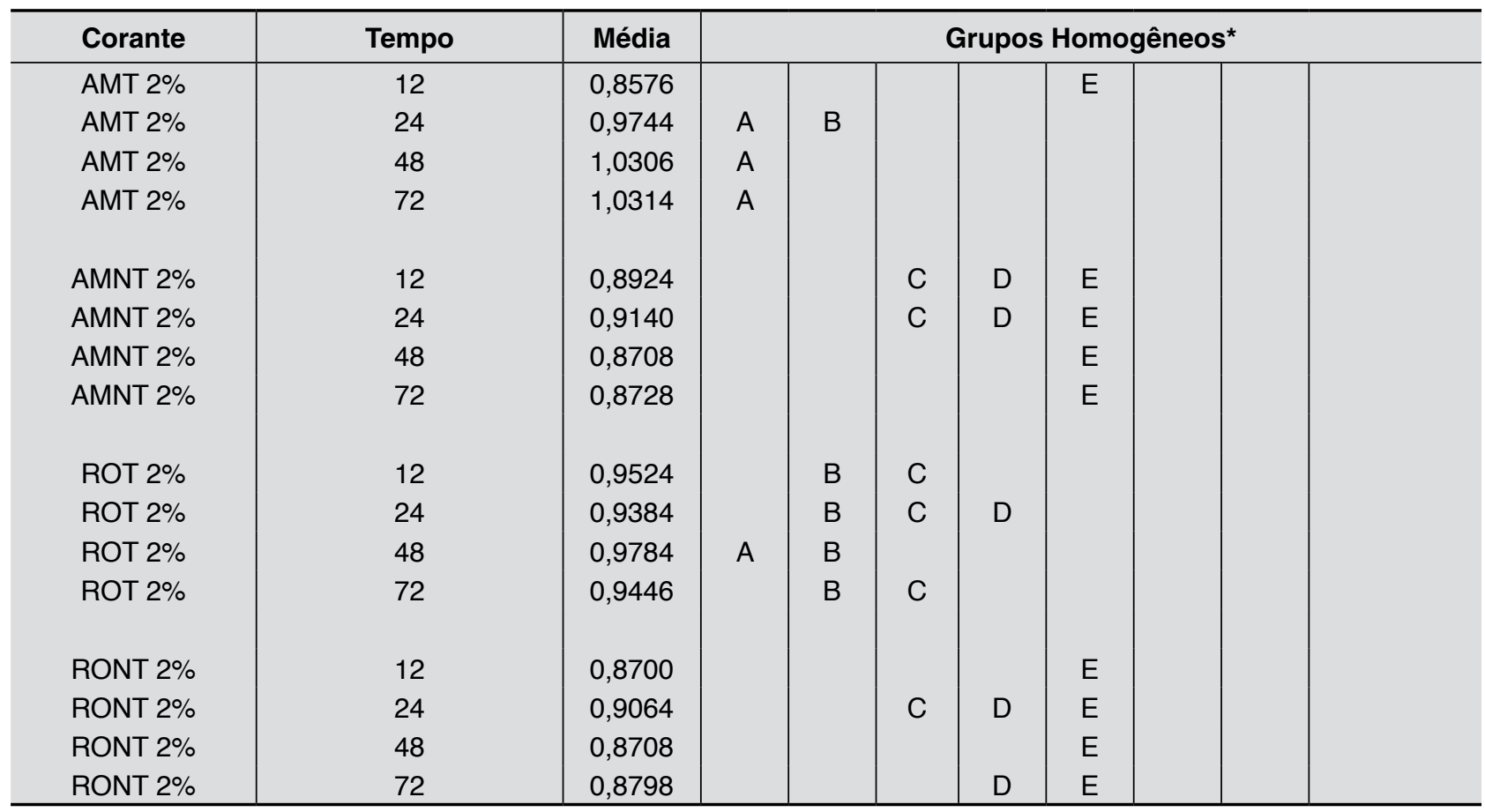

* Médias seguidas de letras iguais na mesma coluna não diferem estatisticamente

Tabela 5 - Teste de comparação múltipla de Tukey (5\%) para as diferentes condições experimentais dos dados da densidade óptica após a imersão dos espécimes do cimento de Portland

\begin{tabular}{|c|c|c|c|c|c|c|c|c|}
\hline \multirow{2}{*}{$\begin{array}{l}\text { Corante } \\
\text { AMT } 2 \%\end{array}$} & \multirow{2}{*}{$\begin{array}{c}\text { Tempo } \\
12\end{array}$} & \multirow{2}{*}{$\begin{array}{l}\text { Média } \\
1,0038\end{array}$} & \multicolumn{6}{|c|}{ Grupos Homogêneos* } \\
\hline & & & $A$ & $B$ & & & & \\
\hline AMT $2 \%$ & 24 & 1,0346 & $A$ & & & & & \\
\hline AMT 2\% & 48 & 1,0440 & $A$ & & & & & \\
\hline AMT 2\% & 72 & 1,0556 & A & & & & & \\
\hline AMNT 2\% & 12 & 0,8912 & & & $\mathrm{C}$ & $\mathrm{D}$ & $E$ & $\mathrm{~F}$ \\
\hline AMNT 2\% & 24 & 0,9030 & & & C & $\mathrm{D}$ & $E$ & $\mathrm{~F}$ \\
\hline AMNT $2 \%$ & 48 & 0,8900 & & & & $\mathrm{D}$ & $E$ & $\mathrm{~F}$ \\
\hline AMNT 2\% & 72 & 0,8942 & & & C & $\mathrm{D}$ & $\mathrm{E}$ & $\mathrm{F}$ \\
\hline ROT $2 \%$ & 12 & 0,9524 & & B & C & & & \\
\hline ROT 2\% & 24 & 0,9450 & & B & C & $\mathrm{D}$ & $E$ & \\
\hline ROT 2\% & 48 & 0,9514 & & B & $\mathrm{C}$ & $\mathrm{D}$ & & \\
\hline ROT 2\% & 72 & 1,0336 & $A$ & & & & & \\
\hline RONT 2\% & 12 & 0,9364 & & & C & $\mathrm{D}$ & $E$ & $F$ \\
\hline RONT 2\% & 24 & 0,9148 & & & C & $\mathrm{D}$ & $E$ & $\mathrm{~F}$ \\
\hline RONT 2\% & 48 & 0,8802 & & & & & & $\mathrm{~F}$ \\
\hline RONT 2\% & 72 & 0,8850 & & & & & E & $\mathrm{F}$ \\
\hline
\end{tabular}

* Médias seguidas de letras iguais na mesma coluna não diferem estatisticamente 


\section{Dıscussão}

A análise da densidade óptica de uma substância pelo espectrofotômetro mostra a provável reação entre a solução e os materiais em teste. A diminuição nos valores da densidade óptica pode indicar uma redução na concentração da solução, enquanto o aumento nos valores da densidade óptica pode indicar um aumento na concentração. $\mathrm{O}$ aumento dos valores da densidade óptica pode indicar uma interação química na estrutura da solução devido à solubilidade das partículas dos materiais em teste e da transferência destas para a solução ${ }^{14}$.

$\mathrm{Na}$ análise dos dados obtidos (Tabela 1), sem a imersão de qualquer espécime, verifica-se que as soluções corantes apresentaram valores semelhantes de densidade óptica nos diferentes períodos de tempo de análise (período 1 e 2). Este comportamento estável dos dados de densidade óptica, sem a imersão dos espécimes, mostrou que o tempo não foi um fator que pudesse alterar o comportamento das soluções ao longo do experimento.

$\mathrm{Na}$ Tabela 2 podem ser observados a média e o desvio padrão da densidade óptica verificada após a imersão dos espécimes nas diferentes soluções corantes. Os resultados da análise de variância mostraram haver diferenças significativas no comportamento dos corantes em relação ao tempo e ao tipo de material $(\mathrm{p}<0,05)$ (Tabela 3).

Na Tabela 4 verifica-se que houve diferença estatisticamente significativa nos valores da densidade óptica do corante azul de metileno $2 \%$ tamponado após a imersão dos espécimes de cimento Sealer 26 nos períodos de 24, 48 e 72 horas em relação do período de 12 horas. Na Tabela 5 verifica-se que houve diferença estatisticamente significativa nos valores da densidade óptica do corante rodamina B 2\% tamponado após a imersão dos espécimes de cimento Portland nos períodos de 12, 24 e 48 horas em relação do período de 72 horas.

Wu et al. ${ }^{24}$ (1998) investigaram a exposição de diferentes materiais de selamento à solução de azul de metileno, por meio da análise da densidade óptica da solução corante. Os autores verificaram que a solução de azul de metileno $1 \% \mathrm{com} \mathrm{pH}$ ajustado a sete pode ser descolorida significativamente pelo agregado de trióxido de mineral e pelo hidróxido de cálcio. De maneira semelhante, Pizzolato et al. ${ }^{15}$ (2002) verificaram a descoloração da rodamina $\mathrm{B}$, do verde brilhante $\mathrm{e}$ do cristal violeta pela ação do hipoclorito de sódio, analisando a absorbância relativa no comprimento de onda de maior absorbância $\left(\lambda_{\text {máx }}\right)$ de cada composto, utilizando um espectrofotômetro e um caminho óptico (cubeta) de $1 \mathrm{~cm}$.

Observando-se a Figura 2 verifica-se que os valores da densidade óptica das soluções corantes após a imersão dos espécimes de Sealer 26 por 12 horas foram inferiores aos observados sem a imersão. Além disso, na solução de azul de metileno $2 \%$ não tamponada, rodamina B $2 \%$ tamponada e na rodamina B $2 \%$ não tamponada, pode-se verificar que os valores da densidade óptica das soluções corantes após a imersão dos espécimes permaneceram estáveis por 12, 24, 48 e 72 horas (Tabela 4), apresentando valores inferiores aos de cada corante antes da imersão dos espécimes. Resultados semelhantes foram verificados por Wu et al. ${ }^{24}$ (1998), que verificaram a descoloração da solução de azul de metileno $1 \% \mathrm{com}$ pH ajustado a sete pelo agregado de trióxido de mineral e pelo hidróxido de cálcio. Segundo Wu et al. ${ }^{24}$ (1998), a extensão da penetração do corante azul de metileno em estudos de infiltração pode não revelar a total extensão da falha ao longo da obturação, uma vez que essa solução pode ser significativamente descolorida por alguns materiais dentários.

Observando-se a Figura 2 verifica-se aumento nos valores da densidade óptica da solução de azul de metileno $2 \%$ tamponada após 24, 48 e 72 horas de imersão dos espécimes de Sealer 26. No presente estudo, a imersão dos espécimes nas soluções corantes tamponadas e não tamponadas, por diferentes períodos de tempo, pode ter promovido a solubilização dos mesmos. Ainda, os espécimes de Sealer 26 foram preparados com proporção de pó:líquido diferente da recomendada para a obturação do sistema de canais radiculares, sendo utilizada a proporção preconizada por Tanomaru et al. ${ }^{18}$ (1998) para emprego em cirurgias parendodônticas.

De acordo com Oztan et al..$^{14}$ (2001), a análise da densidade óptica de uma substância pelo espectrofotômetro mostra a provável reação entre a solução e os materiais em teste. $\mathrm{O}$ aumento dos valores da densidade óptica pode indicar uma interação química na estrutura da solução devido à solubilidade das partículas dos materiais em teste e da transferência destas para a solução. Logo, neste estudo, o aumento dos valores da densidade óptica da solução de azul de metileno $2 \%$ tamponada após 24, 48 e 72 horas de imersão de espécimes de Sealer 26 pode ser devido a presença de partículas deste material na solução corante. 
Observando-se a Figura 2 verifica-se aumento dos valores da densidade óptica da solução de azul de metileno $2 \%$ tamponada e da solução de rodamina B 2\% tamponada após a imersão de espécimes do cimento de Portland. Segundo Mehta \& Monteiro ${ }^{13}$ (1994), qualquer meio com $\mathrm{pH}$ menor que 12,5 pode ser qualificado como agressivo ao cimento de Portland, porque a redução da alcalinidade do fluido dos poros levaria, no final, a uma desestabilização dos produtos cimentíceos de hidratação. A presença de partículas dos materiais em teste pode ter promovido o aumento dos valores da densidade óptica das soluções corantes nos diferentes períodos de tempo de análise.

Observando-se a Figura 2 verifica-se que os valores da densidade óptica das soluções corantes não tamponadas após a imersão dos espécimes de cimento de Portland por 12 horas foram inferiores aos observados sem a imersão. Wu et al. ${ }^{24}$ (1998) verificaram a descoloração da solução de azul de metileno $1 \% \mathrm{com}$ pH ajustado a sete pelo agregado de trióxido de mineral e pelo hidróxido de cálcio. Segundo Mehta \& Monteiro ${ }^{13}$ (2004), a matriz do cimento de Portland desenvolve um $\mathrm{pH}$ por volta de 13,5 devido a liberação de $\mathrm{NaOH}$ e $\mathrm{KOH}$. Além disso, com o desenvolvimento da hidratação, a reserva de hidróxido de cálcio formada tamponará $\mathrm{o} \mathrm{pH}$ a um valor por volta de 12,4 , que pode levar a descoloração da solução de azul de metileno pela formação do thional ${ }^{4,5}$.

De acordo com Wu \& Wesselink ${ }^{23}$ (1993), a solução aquosa de azul de metileno, freqüentemente utilizada nos estudos de infiltração por corante, é ácida e pode desmineralizar a dentina, podendo provocar variações na avaliação da infiltração. $\mathrm{O}$ tamponamento do $\mathrm{pH}$ da solução corante a valores maiores que cinco evitaria a desmineralização da estrutura dentinária, evitando o aumento da penetração do corante nos estudos de infiltração $0^{17,23}$.
Em alguns estudos onde a solução de rodamina B foi utilizada como traçador em testes de microinfiltração de retrobturações realizadas com MTA, os autores verificaram que o corante foi capaz de revelar falhas no selamento ${ }^{10,11}$. Pizzolato et al. ${ }^{15}$ (2002) verificaram que a rodamina B apresentou maior resistência à descoloração pelo hipoclorito de sódio $2 \%$ e pela exposição solar em frascos de borosilcato de sódio em relação ao verde brilhante e do cristal violeta.

De acordo com os resultados de Starkey et al. ${ }^{17}$ (1993), Wu et al..$^{24}$ (1998), Oztan et al. ${ }^{14}(2001)$ e os verificados no presente estudo, alguns materiais podem interagir com os corantes empregados no estudo da infiltração marginal em diferentes períodos de tempo, podendo alterar a difusão ou a coloração do agente traçador, influenciando a avaliação dos resultados nos estudos de infiltração marginal.

\section{Conclusão}

a) a imersão dos espécimes de Sealer 26 promoveu redução na densidade óptica de todas as soluções corantes, exceto do azul de metileno $2 \%$ tamponado;

b) a imersão dos espécimes de cimento Portland promoveu redução na densidade óptica das soluções não tamponadas;

c) dentre as soluções corantes analisadas, a rodamina B $2 \%$ tamponada apresentou comportamento mais estável quanto aos valores de densidade óptica até o período de avaliação de 48 horas, o que sugere uma melhor capacidade de evidenciar falhas no selamento marginal.

\section{Abstract}

The purpose of this study was to evaluate the optical density of $2 \%$ methylene blue dye solution buffered and unbuffered, and $2 \%$ rhodamine dye solution buffered and unbuffered, before and after the immersion of endodontic retrofilling materials in different time intervals. The dye solutions were evaluated without the immersion of the specimens at $0,12,24$, 48 and 72 hours. Eighty specimens of Sealer 26 cement and eighty specimens of Portland cement were immersed in 0,7 $m L$ of each dye solution at 12, 24, 48 and 72 hours. The optical density was evaluated through a spectrophotometer at $596 \mathrm{~nm}$ for methylene blue dye solution and at 566nm for rhodamine dye solution. The optical density data was analysed using three-way ANOVA followed by Tukey's test. The results demonstrated that there were statistical significant difference in optical density values for $2 \%$ methylene blue dye solution after the immersion of Sealer 26 cement at the 
time interval of 24, 48 e 72 hours when compared to the 12 hours interval. The results demonstrated that there were statistical significant difference in optical density values for $2 \%$ rhodamine dye solution after the immersion of Portland cement at the time interval of 12, 24 and 48 hours when compared to the 72 hours interval. The unbuffered solutions presented the lowest optical density values. The $2 \%$ rhodamine buffered dye solution presented values of optical density more stable at the time interval of 48 hours.

\section{UNITERMS}

Dyes; optical density; dental material; retrofilling material.

\section{REFERÊNCIAS}

1. Abedi HR, Ingle JI. Mineral trioxide aggregate: a review of a new cement. J Calif Dent Assoc 1995 Dec.; 23 (12): 36-39.

2. Abdullah D, Ford TR, Papaioannou S, Nicholson J, McDonald F. An evaluation of accelerated Portland cement as a restorative material. Biomaterials 2002 Oct.; 23 (19): 4001-10.

3. Assis NMSP. Avaliação do selamento de ápices radiculares preparados com ultra-som e brocas e retrobturados com diferentes materiais mediante infiltração marginal por corante. [dissertação] São José dos Campos: Faculdade de Odontologia de São José dos Campos, Universidade Estadual Paulista; 2001..

4. Bernabé PFE, Holland R. MTA e cimento Portland: considerações sobre as propriedades físicas, químicas e biológicas. In: Cardoso RJA, Machado MEL. Odontologia arte e conhecimento, São Paulo: Artes Médicas; 2003: 225-4.

5. Coffey S. Heterocyclic compounds. In: Rodd's chemistry of carbon compounds: a modern comprehensive treatise. 2.ed. Amsterdam: Elsevier Scientific Publishing; 1978: 531-32.

6. Duarte MAH, Weckwerth PH, Kuga MC, Weckwerth ACV. Avaliação da contaminação do MTA Angelus e do cimento de Portland. J Bras Clin Odontol Int 2002 mar./abr.; 6 (32): 155-57.

7. Estrela C, Bammann LL, Estrela CR, Silva RS, Pécora JD. Antimicrobial and chemical study of MTA, Portland cement, calcium hydroxide paste, Sealapex and Dycal. Braz Dent J 2000; 11 (1):3-9.

8. ESTRELA, C. Ciência endodôntica. São Paulo: Artes Médicas; 2004

9. Gomes APM. Avaliação do selamento de ápices radiculares retrobturados ou recobertos com diferentes materiais mediante infiltração marginal por corante e análise da interface em microscopia eletrônica de varredura. [tese] São José dos Campos - Faculdade de Odontologia de São José dos Campos; 1997.

10. Gonçalves SB, Bramante CM. Avaliação in vitro da capacidade seladora do super EBA e do MTA em quatro técnicas de obturação retrógrada. Rev Fac Odontol Bauru 2002; 10 (3): 170-78.

11. Kubo CH. Avaliação do selamento de ápices radiculares tratados com diferentes agentes desmineralizantes e retrobturados com PRO ROOT - MTA, mediante infiltração marginal por corante. [dissertação] São José dos Campos - Faculdade de Odontologia de São José dos Campos, Universidade Estadual Paulista; 2001.

12. Leonardo MR. Endodontia: tratamento de canais radiculares: princípios técnicos e biológicos. São Paulo: Artes Médicas, 2005.

13. Mehta PK, Monteiro PJM. Concreto: estrutura, propriedades e materiais. São Paulo: Pini; 1994.

14. Öztan MD, Özgey E, Zaimoglu L, Erk N. The effect of various root canal sealers on India ink and different concentrations of methylene blue solutions. J Oral Science 2001; 43 (4): 245-48.

15. Pizzolato TM, Carissimi E, Machado EL, Schneider IA. Colour removal with $\mathrm{NaOCl}$ of dye wastewater from and agate-processing plant in Rio Grande do Sul, Brazil. Int J Mineral Processing 2002; 65: 203-11.

16. Souza EM. Influência dos corantes azul de metileno a $2 \%$ e rodamina B a $2 \%$ na determinação da capacidade seladora apical de diferentes cimentos endodônticos.[dissertação] Araraquara - Faculdade de Odontologia de Araraquara, Universidade Estadual Paulista; 2004.

17. Starkey DL, Anderson RW, Pashley DH. An evaluation of the effect of methylene blue dye pH on apical leakage. J Endod 1993 Sept.; 19 (9): 435-39.

18. Tanomaru Filho M, Bronzi ES, Wilhelmsen NSW, Ogata M. Capacidade seladora de diferentes cimentos endodônticos em obturações retrógradas. Rev Fac Odontol Lins 1998 jan./jun.; 11 (1): 58-61.

19. Torabinejad M, Watson TF, Pitt Ford TR. Sealing ability of a mineral trioxide aggregate when used as a root end filling material. J Endod 1993 Dec.; 19 (12): 591-95.

20. Torabinejad M, Smith PW, Kettering JD, Pitt Ford TR. Comparative investigation of marginal adaptation of mineral trioxide aggregate and other commonly used root-end filling materials. J Endod 1995 June; 21 (6):295-99

21. Torabinejad M, Hong CU, McDonald F, Pitt Ford TR. Physical and chemical properties of a new root-end filling material. J Endod 1995 July; 21 (7): 349-53.

22. Wucherpfenning AL, Green DB. Mineral trioxide vs. Portland Cement: two compatible filling materials. J Endod 1999 Apr.; 25 (4): 308 (Abstract PR40).

23. Wu MK, Wesselink PR. Endodontic leakage studies reconsidered. Part I. Methodology, application and relevance. Int Endod J 1993; 26 : 37-52.

24. Wu MK, Kontakiotis EG, Wesselink PR. Decoloration of $1 \%$ methylene blue solution in contact with dental filling materials. J Dent 1998; 26 : 585-89.

25. Youngson CC, Jones JCG, Manogue M, Smith IS. In vitro dentinal penetration by tracers used in microleakage studies. Int Endod J 1998; 31 (2): .90-9.
Recebido em: 15/06/07 Aprovado em: 23/08/07

Endereço para correspondência: Neuza Maria Souza Picorelli Assis e-mail: neuzapicorelli@terra.com.br

Rua Delfim Moreira, 199/1100.

Centro - Juiz de Fora - MG CEP: $36010-570$ 